\title{
How do sock ply changes affect residual-limb fluid volume in people with transtibial amputation?
}

\author{
Joan E. Sanders, PhD; ${ }^{1-2^{*}}$ Daniel S. Harrison, BSEE; ${ }^{1}$ Katheryn J. Allyn, CPO; $^{1}$ Timothy R. Myers, MME; $^{1}$ \\ Marcia A. Ciol, PhD; ${ }^{2}$ Elaine C. Tsai, $\mathbf{M D}^{3}$ \\ Departments of ${ }^{1}$ Bioengineering and ${ }^{2}$ Rehabilitation Medicine, University of Washington, Seattle, WA; ${ }^{3}$ Department of \\ Veterans Affairs Puget Sound Health Care System, Seattle, WA
}

\begin{abstract}
The purpose of this research was to investigate the influence of sock addition and removal on residual-limb fluid volume in people using prosthetic limbs. We used bioimpedance analysis to measure residual-limb extracellular fluid volume on 28 transtibial amputee subjects during $30 \mathrm{~min}$ test sessions. Upon addition of a one-ply polyester sock, residual-limb fluid volume changes ranged from $-4.0 \%$ to $0.8 \%$ (mean $-0.9+/-$ $1.3 \%$ ) of the initial limb fluid volume. Changes for sock removal ranged from $-1.2 \%$ to $2.8 \%$ (mean $0.5+/-0.8 \%$ ). Subjects who reduced in fluid volume with both addition and removal of a sock and subjects with high positive ratios between the fluidvolume loss upon sock addition and gain upon sock removal (high add/remove [AR] ratios) tended to have arterial disease, were obese, and were smokers. Subjects with low positive AR ratios, subjects who increased in fluid volume both with sock addition and removal, and a single subject who increased in fluid volume with sock addition and decreased with sock removal tended to be nonsmokers and either individuals in good health without complications or individuals without arterial problems. Results are relevant for the anticipation of limb volume changes during prosthetic fitting and toward the design of adjustablesocket technologies.
\end{abstract}

Key words: accommodation, amputee, bioimpedance, fluid volume, interface stress, prosthesis, residual-limb volume, socket fitting, sock ply, transtibial.

\section{INTRODUCTION}

For individuals using lower-limb prostheses, daily changes in the volume of the residual limb can cause discomfort and pain. Changes in limb volume affect how the prosthesis fits [1] and how mechanical pressures and shear stresses are delivered to the residual limb [2]. Typically, when a patient's residual limb decreases in volume more stress is applied at bony prominences, such as the anterior distal tibia and the fibular head, and this elevated stress may cause pain and increase the risk of breakdown and injury. When a patient's residual limb increases in volume, blood flow may be obstructed, limiting nutrient delivery and causing a buildup of cell waste products in the residuum. Residual-limb volume changes also affect suspension and how much the limb pistons (moves up and down) in the socket during ambulation. Pistoning induces frictional stress, i.e., rubbing with slipping between the limb and socket, which induces injury faster than shear stress, i.e., rubbing without slipping [3].

\footnotetext{
Abbreviations: $\mathrm{ABI}=$ ankle brachial index, $\mathrm{AR}=\mathrm{add} /$ remove, ASGP = ambulatory strain-gauge plethysmography, $\mathrm{BMI}=$ body mass index.

*Address all correspondence to Joan E. Sanders, PhD; University of Washington-Bioengineering, 355061, 3720 15th Ave NE, University of Washington, Seattle, WA 98195; 206221-5872; fax: 206-685-3300.

Email: jsanders@u.washington.edu

http://dx.doi.org/10.1682/JRRD.2011.02.0022
} 
Adding or removing socks is a common way for people using prostheses to accommodate daily residual-limb volume changes. For many people with lower-limb amputation, their limb volume is greatest early in the day after rising. After walking with the prosthesis, they may experience a reduction in limb volume. Volume reduction can continue through the day as a result of the continual stresses applied by the prosthetic socket during ambulation. Individuals who use a shrinker (an elastic sock worn on the residuum) at night or individuals with venous vascular complications may experience the opposite trend, an increase in limb volume (edema) during the day. These individuals may reach their maximal limb volume in the evening. Whether experiencing limb volume increases or decreases over the day, people with limb amputation often carry extra socks with them that they add or remove in an effort to keep an adequate socket fit despite residual-limb volume changes.

While adding or removing socks adjusts the fit of the socket, these adjustments cause a change in the socket pressures and shear stresses applied to the residual limb that in turn might affect limb volume. Adding socks may increase interstitial fluid pressure, decreasing the person's limb volume even further. Removing socks may induce the opposite effect and increase residual-limb volume. However, limb volume regulation is under complex physiological control, and it is unknown if adding and removing socks actually induce these changes. It is also unknown if the residual-limb fluid volume reduction from sock addition is comparable to the residual-limb fluid volume increase from subsequent sock removal.

The purpose of this study was to investigate the short-term effects of adding and removing socks on residual-limb fluid volume in people with transtibial amputation. We used bioimpedance analysis for the fluid volume assessment to answer the questions, Is residuallimb fluid volume affected by adding or removing socks, and are the changes for adding a sock of comparable magnitude but opposite in direction from those for removing a sock? We also investigated how the results were related to the participant's health.

\section{METHODS}

\section{Subjects}

Subjects were included if they had a transtibial amputation that was performed at least 12 months before enrollment in the study and their residual limb was at least $9 \mathrm{~cm}$ in length from the mid-patellar-tendon to the distal end. To participate, subjects were required to have a definitive prosthesis that they wore at least 5 hours per day. Their prosthesis needed to fit properly and function safely, as assessed by the research practitioner. Using a flexible probe, the practitioner assessed fit and adjusted sock ply at the outset of the session to ensure the residual limb was adequately supported in the proximal and central regions. Subjects were not included if they could not ambulate with a prosthesis for $10 \mathrm{~min}$ indoors on a level walkway or if they could not shift from a vertical to horizontal posture within $30 \mathrm{~s}$ (necessary for vascular tests). Subjects with current skin breakdown were excluded from the study. If the subject had bilateral amputation, we used the residual limb that met the inclusion criteria. If both residual limbs met the inclusion criteria, then we considered several factors in selecting the limb to monitor: limb length, amount of scar tissue, and ease of monitoring. The limb expected to produce the strongest, most reliable bioimpedance signal was selected.

\section{Instrumentation}

A multifrequency bioimpedance analyzer (XiTRON Hydra 4200, ImpediMed; San Diego, California) that we modified for the purpose of testing people with transtibial amputation was used for assessing residual-limb extracellular fluid volume change. To ensure minimal disruption to signal quality during the subject's ambulation, we created a custom four-pin Delrin connector that accommodated gold-plated pins (WPI Viking, Cooper Interconnect; Chelsea, Massachusetts) to attach four 28-gauge insulated lead wires from the XiTRON instrument cable to the electrodes. To strain-relieve and mechanically stabilize the wire-to-electrode connection after it was soldered together, we looped the wire onto the tab of the electrode, placed a polystyrene disk $(9 \mathrm{~mm}$ diameter, $0.25 \mathrm{~mm}$ thickness) over the connection, and wrapped it with a single layer of vinyl electrical tape (Scotch super vinyl electrical tape 88, 3M; St. Paul, Minnesota). A 9 × $26 \mathrm{~mm}$ section was cut from the adhesive part of a BandAid (Johnson \& Johnson; New Brunswick, New Jersey), and its outer surface was glued (Skin-Bond Cement, Smith \& Nephew; London, United Kingdom) onto the outside of the electrical tape over the tab so that the Band-Aid's sticky surface was exposed to the skin. The purpose of the Band-Aid was to ensure that both the tab stuck to the skin and the tab-solder connection was not 
excessively strained and damaged during use. These efforts substantially enhanced signal-to-noise ratio and decreased failure at the wire-to-electrode connection. The largest source of error in the bioimpedance measurement was bit quantification. The smallest fluid volume change that could be resolved depended on the subject's limb fluid volume but was not larger than 0.2 percent for all subjects.

Bioimpedance has been used extensively in other areas of biomedicine, including body composition/body fat analysis [4-9] and assessment of fluid imbalance in hemodialysis patients [10-13]. It has been shown to correlate well with other measurement techniques, including deuterium oxide and bromide dilution [14-17], as well as with magnetic resonance imaging [18]. In this study, we defined "residual-limb fluid volume change" as the extracellular fluid volume change measured using the multifrequency bioimpedance analysis system. Only extracellular fluid was considered in the analysis because of its likely strong influence on short-term limb fluid volume change [19].

To conduct bioimpedance analysis on transtibial amputee subjects, four electrodes were placed on the residual limb (Figure 1). The outer pair was currentinjecting, while the inner pair was voltage-sensing. Current was applied to the current-injecting electrodes at between 50 and $700 \mu \mathrm{A}$ at 50 frequencies between $5 \mathrm{kHz}$ and $1 \mathrm{MHz}$ while voltage (amplitude and phase) was measured with the voltage-sensing electrodes. The system recorded resistance, reactance, impedance, and phase once per second for all 50 frequencies.

\section{Protocol}

Subjects were asked to refrain from drinking coffee or alcohol before coming to the laboratory on the day of testing. We started tests between the hours of 9:00 a.m. and 1:30 p.m. Each subject's weight and height were recorded, and body mass index (BMI) was calculated as the quotient of the mass and the square of the height (kilograms per meter squared) [20]. The subject wore the prosthesis while mass was recorded to offset BMI measurement underestimates that occur when people with transtibial amputation do not wear their prosthesis for measurement [21]. The research practitioner queried the subjects at the outset of the test session to determine if the socket was comfortable. Sock ply was adjusted if needed. Then the subject walked briefly on a treadmill (Quinton Clubtrack, Cardiac Science; Bothell, Washington) to set a normal self-selected walking speed. The sub- ject then sat in a chair and rested comfortably for $20 \mathrm{~min}$ with the prosthesis donned while the research practitioner queried the subject on recent medical history and perceived socket comfort. Vascular and cardiovascular health and disease histories were recorded. The subject then doffed the prosthesis, and the research practitioner inspected the residual limb to ensure there were no sores or injury. The skin was prepared for bioimpedance measurement by gently rubbing the regions where the electrodes were to be placed with sandpaper (Red Dot Trace Prep 2236, 3M) [22]. A thin layer of coupling gel (Couplant D, GE Panametrics; Fairfield, Connecticut) was applied to the skin side of each electrode before fixing it to the skin. All electrodes were placed parallel to each other on the lateral posterior region of the residual limb (Figure 1). This region was used so that the bone did not interfere with the electrical current flow through the soft tissues. In addition, when positioned on the lateral posterior surface, the electrodes were comfortable to the subjects. The voltage-sensing electrodes were positioned

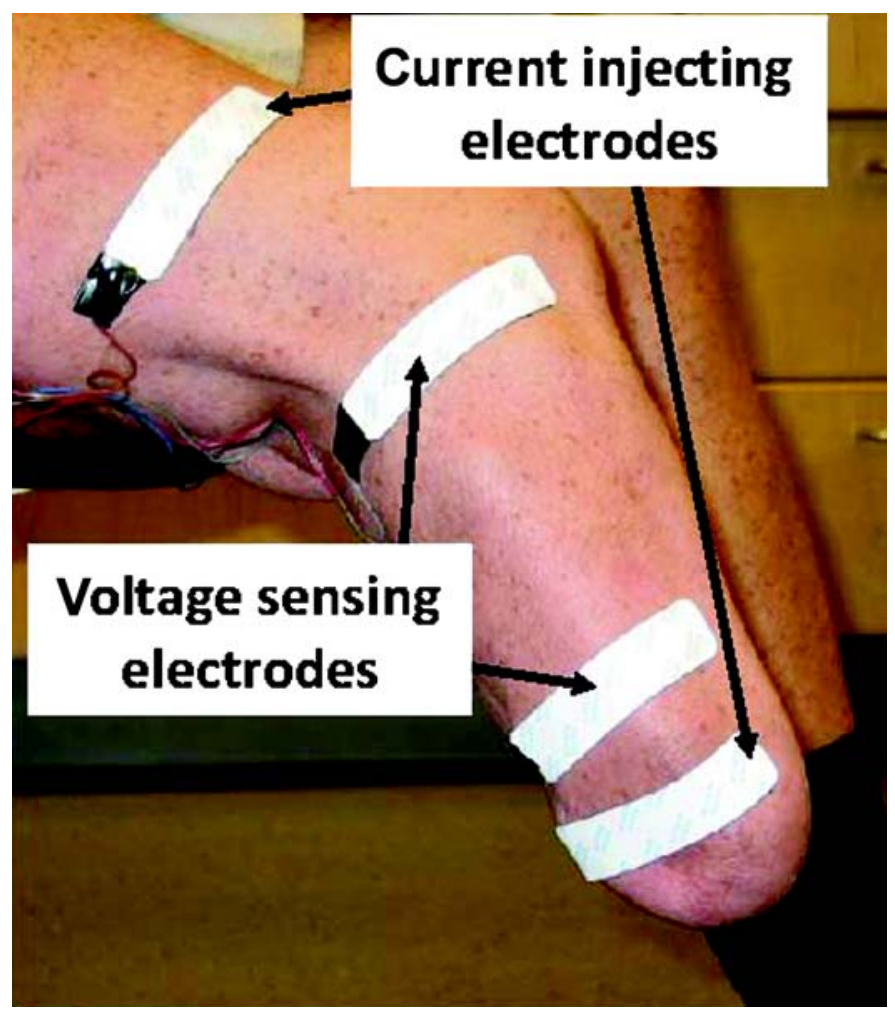

Figure 1.

Electrode placement for bioimpedance testing. Outer two electrodes injected current, while inner two electrodes sensed voltage. 
to maximize the segment measured below the knee but not to interfere with bony prominences. The proximal voltage-sensing electrode was placed at the level of the patellar tendon, proximal to the fibular head. Voltagesensing electrodes were not placed above the level of the patellar tendon because knee flexion would have distorted the measurement. The distal current-injecting electrode was placed as far distally as possible but still on the relatively cylindrical part of the limb. The distal voltagesensing electrode was placed between 3.0 and $5.5 \mathrm{~cm}$ proximal to the distal current-injecting electrode. The proximal current-injecting electrode was placed on the thigh under the liner or suspension sleeve. Tegaderm (3M) was used to strain-relieve the wires extending from the electrodes out the socket brim. Once the electrodes were applied, residual-limb circumference was measured at the levels of the voltage-sensing electrodes and data collection was initiated.

The subject donned the prosthesis and sat still without talking for 2 min with the prosthetic foot supported on the floor. Care was taken to ensure good sitting posture since too much knee flexion occludes blood flow and too much extension causes a slouching posture. To achieve good sitting posture, the subject's knee was typically in approximately $100^{\circ}$ of extension. The subject then walked on the treadmill for 3 min at the normal selfselected walking speed established at the outset of the session. Next, the subject stood for approximately $12 \mathrm{~s}$ with equal weight bearing on a $6.4 \mathrm{~cm}$ high platform with an electronic weight scale (349KLX Health-O-Meter, Pelstar; Alsip, Illinois) embedded within it so that the top of the scale was flush with the platform surface. The scale was monitored at a $2 \mathrm{~Hz}$ sampling rate. If the weight on the scale deviated by more than 10 percent of half the subject's body weight, then the subject was instructed by the research practitioner to shift his or her weight to the appropriate leg to achieve equal weightbearing. The subject then sat, doffed the prosthesis, and added a 1-ply sock (1SP1-RG-SH Lightweight Soft Sock, Knit-Rite; Kansas City, Kansas), a process that typically took less than $60 \mathrm{~s}$. According to manufacturer documentation, the sock was 90.6 percent polyester, 5 percent $\mathrm{X}$ STATIC, and 4.4 percent Lycra Spandex. X-STATIC is a proprietary silver-based antimicrobial material (Noble Fiber Technologies, Inc; Scranton, Pennsylvania). Lycra Spandex is a synthetic fiber with high elasticity (Invista; Wichita, Kansas). The sock was added over other socks if other socks were worn, inside the Pelite liner if only a
Pelite liner was worn, and over the outside of the elastomeric liner if only an elastomeric liner was worn. A 1-ply Soft Sock was used because it was considered thin enough to be tolerant to the subjects for the short testing period, but thick enough for its addition to be of clinical relevance. A 1-ply Soft Sock thickness is typical of what a person adds to accommodate diurnal limb fluid volume reduction. The subject then walked on the treadmill again for $3 \mathrm{~min}$, followed by a brief stand under equal weight bearing (12 s). The subject then sat still in a chair without talking for $3 \mathrm{~min}$, followed by a walk on the treadmill for 3 min (still with the added sock). After a brief stand on the scale under equal weight bearing (12 s), the subject sat, doffed the prosthesis, removed the extra sock, and redonned the prosthesis. Again the subject walked on the treadmill for $3 \mathrm{~min}$ and briefly stood on the scale with equal weight bearing (12 s), then sat down and doffed the prosthesis, liner, and socks. The subject sat still for 10 min while bioimpedance data were collected.

During testing, bioimpedance data and data from the electronic scale were viewed in essentially real time $(3 \mathrm{~s}$ delay) at a $0.5 \mathrm{~Hz}$ sampling rate using custom MATLAB (v. 7.10, MathWorks; Natick, Massachusetts) code implemented on a personal computer (Latitude D620, Dell; Round Rock, Texas). The computer acquired the data from the host bioimpedance computer via a USB connection over the internet. The custom MATLAB code processed the bioimpedance data using a Cole model [23], similar to that used by the XiTRON postprocessing program. This display was essential during data collection to verify that the instrumentation was functioning properly and to identify abnormalities during data collection that needed correction, for example the subject sitting in a manner that occluded a major vein in the limb (apparent as a rapid change in limb fluid volume during sitting).

After the session, all data were processed using software supplied by the manufacturer (v.2.2, XiTRON). The algorithm optimized a nonlinear least square error of magnitude and phase to determine extracellular fluid resistance using the Cole model approach described in the literature [23]. The XiTRON algorithm removed data points with large contributions to the least square error. Limitations ensured that less than 25 percent of the data were deleted and that at least one data point was retained for every set of three adjacent frequencies. We then converted the data to extracellular fluid volume using the limb-circumference and segment-length measurements in a well accepted geometric limb model [24], and then 
expressed it as a percentage of the extracellular fluid volume measured at the end of the initial 2 min sit interval with the prosthesis donned.

Residual-limb fluid volume change from sock addition was calculated as the percentage fluid volume measured after the first walk (without the sock) minus that after the second walk (with the sock). Within the $12 \mathrm{~s}$ period after each walk, the first data point at which equal weight bearing was achieved, apparent by visual inspection of the mass measurement from the digital scale, was identified and used in this calculation. Residual-limb fluid volume change from sock removal was calculated as the percentage fluid volume measured after the third walk (with the sock) minus that after the fourth walk (without the sock). To present graphs of limb fluid volume change versus time, we plotted a 10-point moving mean of the percentage change in fluid volume. Since the moving mean filter caused a time delay, the 10-point moving mean was moved back 5 points so as to realign it with the original data. This curve was useful to observe trends over the course of the trial, though it was not used in the analysis calculations described.

To further characterize each subject's health, we conducted a series of vascular assessment tests on a separate day from bioimpedance testing. Tests were consistent with standard clinical protocols to evaluate vascular condition and comprised orthostatic blood pressure, segmental limb pressure, ankle brachial index [25], and ambulatory strain-gauge plethysmography (ASGP) [26]. Tests were conducted 5 to 10 minutes after the subject arrived at the laboratory and sat quietly with the prosthesis donned. The test procedures are described in the following sections.

\section{Orthostatic Blood Pressure}

Each subject's systolic and diastolic blood pressures and heart rate were measured using electronic blood pressure measurement (HEM-775, Omron; Kyoto, Japan). After a baseline measurement was taken with the subject sitting comfortably, additional measurements were taken after the subject rested supine for $3 \mathrm{~min}$, sat upright for $3 \mathrm{~min}$, and stood for $3 \mathrm{~min}$. Systolic blood pressures above $140 \mathrm{~mm} \mathrm{Hg}$ or diastolic above $90 \mathrm{~mm}$ were considered indicative of high blood pressure. Blood pressure changes greater than $20 \mathrm{~mm} \mathrm{Hg}$ for changes in posture were considered abnormal.

\section{Ankle Brachial Index and Segmental Limb Pressure}

The subject rested supine on a hospital bed during testing. Using a commercial segmental limb-pressure measurement system (TD312 Cuff Inflator, MV10 Manifold Selector, and SC12 and SC10 cuffs, Hokanson; Bellevue, Washington) and a Doppler flow meter (MD6 Doppler, Hokanson), we measured blood pressures on the contralateral limb, unless the subject's contralateral limb was amputated above the ankle, and on both limbs above the knee and at the upper thigh. Brachial arm pressure was measured on both upper limbs. The research practitioner identified blood flow changes representing systolic and diastolic pressure thresholds by using the Doppler flow system. At the ankle, either the posterior tibial artery or the anterior tibial artery, whichever had the strongest Doppler signal, was used. Ankle brachial index (ABI) was calculated as the systolic pressure at the ankle on the nonamputated limb (if the subject was not amputated bilaterally) divided by the larger of the two brachial systolic pressures. An ABI greater than 1.3 was considered indicative of a calcified vessel, in which case another vessel in the ankle was pursued and the measurement repeated. An ABI less than 0.9 was considered indicative of more than 50 percent stenosis and thus arterial disease [27-28].

\section{Ambulatory Strain-Gauge Plethysmography}

Measurements were taken from the contralateral limb using a commercial ASGP system (EC6 Plethysmograph, Hokanson). Only unilateral amputee subjects were tested. An elastic band with a strain gauge (indium-gallium) was placed just above the malleolus. The subject stood with the arms extended forward holding onto a waist-high rail for support and then performed 10 deep knee bends (to approximately $60^{\circ}$ of knee flexion). We reduced the number of deep knee bends from the standard 20 described in the literature for ASGP testing [29] because most of our subjects could not conduct more than 10 sequential deep knee bends. The subject then stood still with equal weight bearing for at least $200 \mathrm{~s}$. A recovery time in the strain-gauge data of less than $25 \mathrm{~s}$ was considered to indicate presence of venous insufficiency [26].

\section{RESULTS}

A total of 28 participants with transtibial amputation, 21 male and 7 female, took part in this study. Twenty-five 
had unilateral amputation and three had bilateral amputation. There were 8 subjects at Medicare Functional Classification Level [30] (K-level) K-2 (limited community ambulator), 13 at K-3 (community ambulator), and 7 at K-4 (high level user). Subjects ranged in age from 23 to 72 years, and the mean age was $50 \pm 13$ years. Subject mass ranged from 50.0 to $126.7 \mathrm{~kg}$, with a mean of $87.8 \pm$ $20.6 \mathrm{~kg}$. BMI averaged $28.2 \pm 6.1 \mathrm{~kg} / \mathrm{m}^{2}$. Eight subjects had a BMI between 25 and $30 \mathrm{~kg} / \mathrm{m}^{2}$ (overweight), and nine subjects had a BMI greater than $30 \mathrm{~kg} / \mathrm{m}^{2}$ (obese). Of the 28 participants, 18 had their limb amputation as a result of traumatic injury, 7 for arterial disease, 1 from cancer, 1 from spina bifida, and 1 from Larsson syndrome. Eight subjects currently had diabetes (2 with type 1, 6 with type 2). Four subjects who had their limb amputation as a result of traumatic injury had developed arterial disease since their amputation. Seven of the subjects were current smokers. Two subjects were on dialysis, one had heart arrhythmia, one had congestive heart failure, and one was on lifetime antibiotics because of a citrus allergy that caused lesions on his distal residual limb. Time since amputation ranged from 1 to 49 years, with a mean of $12 \pm 12$ years. All subjects used patellar tendonbearing socket designs except for one subject, who used a total surface-bearing suction socket with a one-way valve at the distal end. Twenty-two participants used elastomeric liners, and of those individuals nineteen used a locking pin and four simultaneously used a Pelite liner with an elastomeric liner. Six participants used Pelite liners without an elastomeric liner. Subject sock ply at the outset of testing averaged $2.8 \pm 2.5$ and ranged from 0 - to 8-ply. We did not measure the thickness of each sock, but instead used subject self-reports of ply values. We began tests between 9:00 a.m. and 12:00 p.m. on 18 subjects and between 12:01 p.m. and 1:30 p.m. on 10 subjects.

An additional subject was tested who met the inclusion criteria, but the protocol for her test session was modified because of concerns the original protocol would induce discomfort. For this subject, the first and fourth walking intervals on the protocol were conducted with an additional sock (3-ply cotton provided by the subject), while the second and third walking intervals were conducted without the additional sock. Thus, the ordering of her sock addition/removal was reversed from the other subjects. The subject was a 60 -year-old female who had her limb amputation (unilateral) as a result of traumatic injury 2 years prior. She was a K-2 ambulator of mass $65.0 \mathrm{~kg}$ and BMI of $24.6 \mathrm{~kg} / \mathrm{m}^{2}$, and she was a former diabetic who did not smoke or have arterial disease. She used a patellar tendon-bearing socket and elastomeric liner with locking pin. She put on a total of eight sock ply in the morning and typically did not add socks during the day. Testing was conducted during morning hours. We present results from this subject as a case study in the "Results" section, separate from the rest of the subjects we tested.

When the sock was added, residual-limb fluid volume decreased for 22 of the 28 subjects and increased for 6 . The percentage fluid volume change from sock addition ranged from -4.0 to 0.8 percent, and the mean change was $-0.9 \pm 1.3$ percent. When the sock was removed, fluid volume increased for 18 of the 28 subjects, decreased for 8 , and did not change for 2 . The percent fluid volume change after sock removal ranged from -1.2 to 2.8 percent, and the mean change was $0.5 \pm 0.8$ percent. Bar graphs of percentage fluid volume change for sock addition and sock removal are shown in Figure 2.

Linear least-squares fits to plots of percentage fluid volume change versus residual-limb cross-sectional area showed a low correlation. The correlation coefficients were 0.03 for sock addition and 0.30 for sock removal. Thus, even though the same size sock (Soft Sock 1-ply) was used in all trials, subjects with smaller residual limbs did not show larger percentage fluid volume changes than subjects with larger limbs or vice versa.

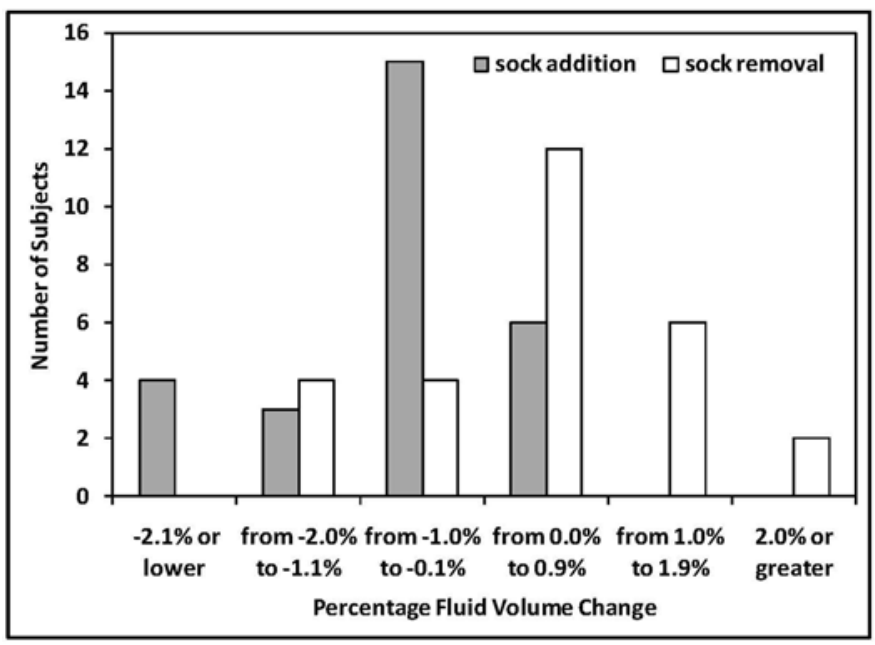

Figure 2.

Percentage residual limb fluid volume change. Number of subjects for different ranges of percentage fluid volume change for sock addition (gray bars) and sock removal (white bars) shown. 
The percentage fluid volume change for sock addition was not of comparable magnitude to that for sock removal. Of the 28 subjects, 18 experienced a greater loss of limb fluid volume after sock addition than gain after sock removal. Ten subjects experienced less loss of limb fluid volume after sock addition than gain after sock removal. The range of the ratio of percentage fluid volume loss from sock addition to percentage fluid volume gain from sock removal, defined here as the AR ratio (add/remove ratio), was -8.3 to 12.0, excluding two subjects whose fluid volume change upon sock removal was 0.0 . The mean ratio was $0.0 \pm 3.7$. The ratio was less than 0.0 for part of the population because some subjects demonstrated fluid volume loss upon both sock addition and sock removal and some showed fluid volume gain upon both sock addition and sock removal. These two groups thus had ratios less than 0.0 . The mean ratio of absolute percentage fluid volume change from sock addition to that from sock removal was $2.3 \pm 2.7$, excluding the two subjects whose fluid volume change upon sock removal was 0.0 (and thus had AR ratios less than -10.0).

We categorized the subjects into four groups based on the directions of their fluid volume changes. Group 1 members $(n=9)$ were defined as subjects who reduced or did not change in fluid volume both when the sock was added and when it was removed. They had a gradual decrease in limb fluid volume over the data collection session (Figure 3(a)). Group 2 members $(n=13)$ were defined as subjects who decreased in fluid volume when the sock was added and increased in fluid volume when it was removed (Figure 3(b)). These subjects demonstrated the expected directions of limb fluid volume change for sock addition and sock removal. Group 3 members $(n=5)$ were defined as subjects who increased in fluid volume both when the sock was added and when it was removed. They had a gradual increase in fluid volume over the session (Figure 3(c)). The group 4 member $(n=1)$ increased in fluid volume when the sock was added and decreased when it was removed. This subject demonstrated the opposite of the expected directions of limb fluid volume change for sock addition and sock removal (Figure 3(d)).

Results from the vascular tests and subject histories showed that 11 subjects had arterial disease and 12 had high blood pressure. Nineteen subjects underwent ASGP testing, and eight had venous insufficiency. Subjects were not tested using ASGP if they had bilateral amputation ( $n=$ $3)$, could not squat $(n=1)$, died or had an above-knee amputation before the tests were completed $(n=2)$, or were not available for the test $(n=3)$. Ten subjects did not have arterial disease, high blood pressure, diabetes, or another major medical disease, and thus were considered in good health. We considered subjects in good health if they had venous insufficiency but not arterial disease, high blood pressure, diabetes, or another major medical condition because the ASGP measurement might have reflected local venous obstruction induced by traumatic injury [31]. ASGP testing was conducted on the contralateral limb rather than the residual limb, and ASGP testing was not conducted on all subjects. Of those 10 subjects in good health, 2 had BMIs between 25 and $30 \mathrm{~kg} / \mathrm{m}^{2}, 1$ had BMI greater than $30 \mathrm{~kg} / \mathrm{m}^{2}$, and 1 was a smoker.

We conducted an exploratory analysis to investigate how subject health status related to bioimpedance results. The percentage fluid volume changes upon sock addition and sock removal were plotted by group (Figure 4), with groups 1 to 4 as defined previously. Within each group, subject data were ordered from highest to lowest AR ratio. The table to the right of the plot identifies if the subject was in good health (no arterial disease, high blood pressure, diabetes, or other major disease) (g), had arterial disease (a), venous insufficiency in the contralateral limb (v), high blood pressure (h), diabetes (d), other major disease (z), a BMI between 25 and $30 \mathrm{~kg} / \mathrm{m}^{2}$ (overweight) (o) or $>30 \mathrm{~kg} / \mathrm{m}^{2}$ (obese) (O), or currently a smoker (s). The last column lists the reason for amputation (R): vascular disease (vd), trauma (t), spina bifida (b), Larsson syndrome (L), or cancer (c).

Results showed noteworthy trends. Subjects in group 2 with high AR ratios (all subjects in group 2 had AR ratios $\geq 0$ ) and subjects in group 1 tended to have arterial disease (Figure 4), and some of them also had venous insufficiency. They also tended to be obese and smokers. Subjects in group 2 with low AR ratios, subjects in group 3 , and the subject in group 4 tended to be nonsmokers and either healthy individuals without complications or individuals without arterial problems. Several subjects in group 2 with low AR ratios and the subject in group 4 had venous insufficiency but not arterial disease. Subjects in group 3 tended to be overweight but not obese. Presence of high blood pressure did not appear to favor any group.

We did not observe trends in the results for Pelite-liner users compared with elastomeric-liner users. AR ratios and fluid volume changes over the session were not distinctly different for the two groups. Similarly, we did not observe a trend relating number of sock ply worn to either AR ratio or to fluid volume change over the session. Linear fits to plots 

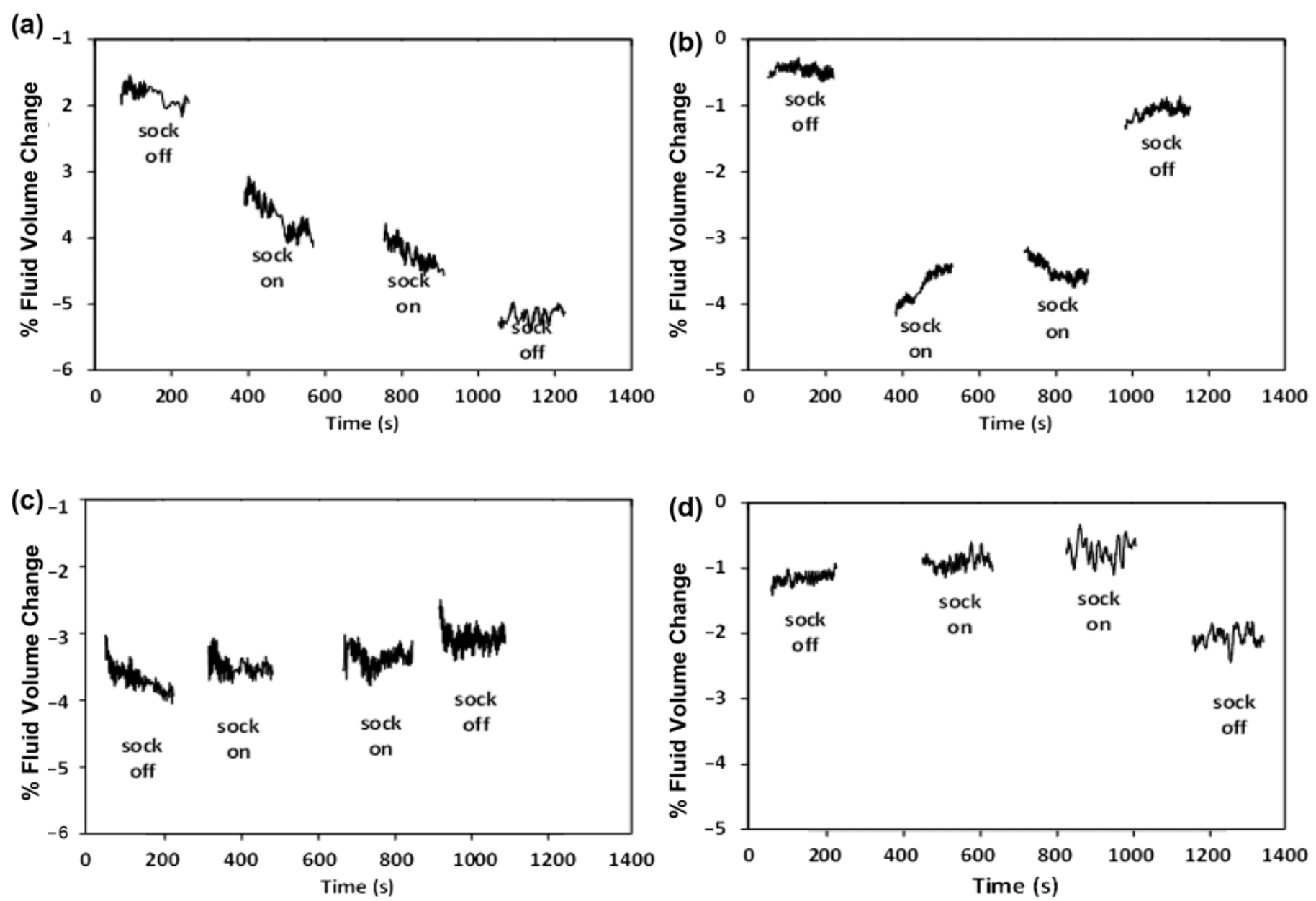

Figure 3.

Exemplary data for one subject from each group are presented. For clarity, only data during walks are shown. Ten-point moving mean plots are presented. (a) Group 1: decrease in limb fluid volume both when sock was added and removed, (b) Group 2: decrease in limb fluid volume when sock was added and increase when it was removed, (c) Group 3: increase in limb fluid volume both when sock was added and removed, (d) Group 4: increase in limb fluid volume when sock was added and decrease when it was removed.

of percentage fluid volume change from adding or removing socks to percentage fluid volume change over the 10 min interval after doffing showed weak correlations. Correlation coefficients were 0.03 for sock addition and 0.30 for sock removal. The subject who was tested using a different protocol from the other subjects (see "Methods" section) underwent a 3.4 percentage fluid volume loss when the sock (3-ply cotton) was added and a 2.1 percent fluid volume gain when the sock was removed. Her AR ratio was 1.6. Her vascular tests revealed that she had high blood pressure and venous insufficiency in the contralateral limb but no other abnormalities. Her results were comparable to other individuals in the upper half of group 2 (those with high AR ratios), except that her percentage fluid volume changes were greater, possibly because she added a 3-ply rather than a 1-ply sock.

\section{DISCUSSION}

This study determined if residual-limb fluid volume was affected by adding or removing socks, and if the changes for adding a sock were of comparable magnitude 


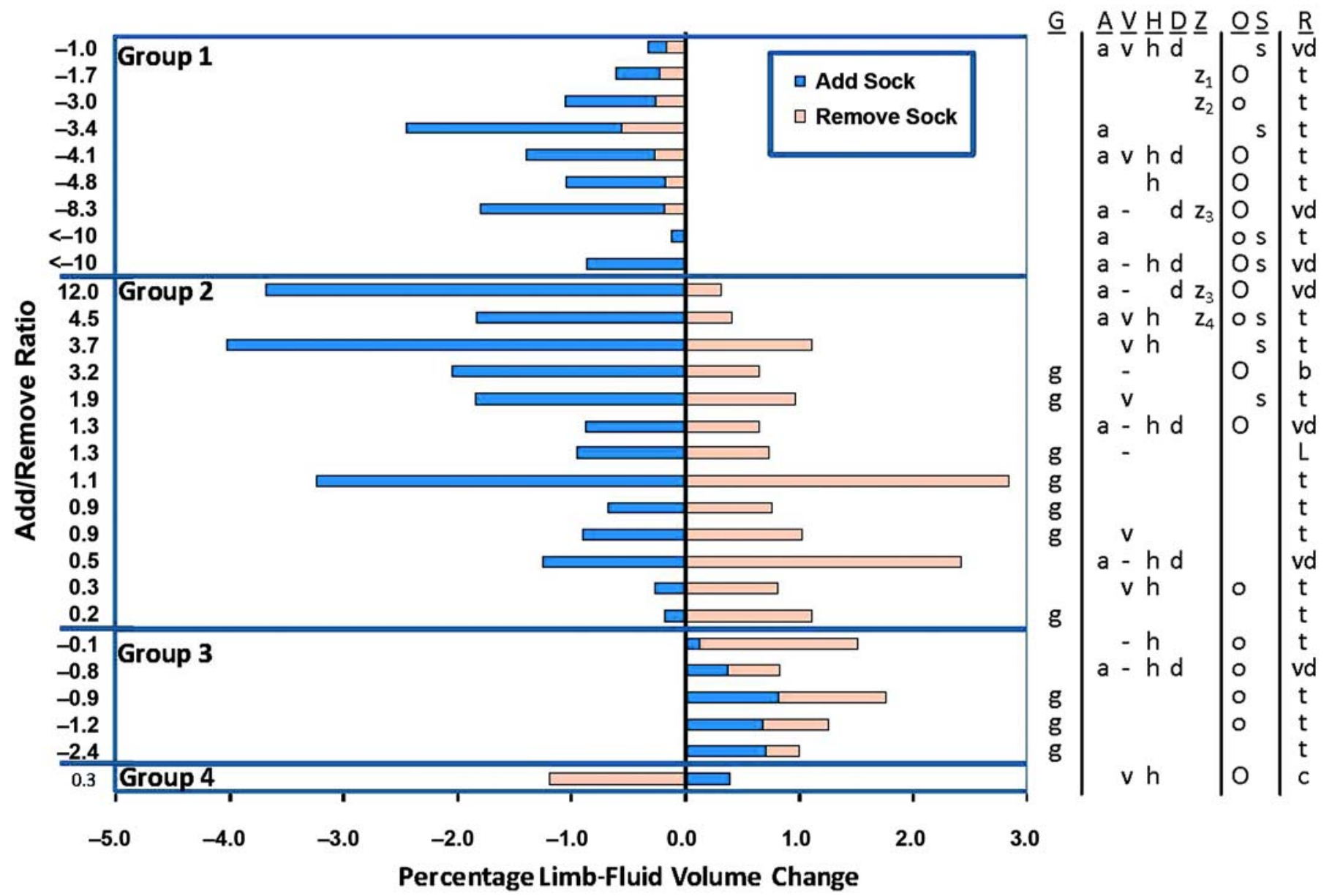

Figure 4.

Subject groups and health status. Percentage limb fluid volume changes for sock addition (blue) and sock removal (orange) shown for all 28 subjects. Groups 1 to 4 were defined based on directions of fluid volume change, as described in text. Numbers to left of graph are add/remove ratios, i.e., quotients of percentage fluid volume change upon sock addition divided by percentage fluid volume change upon sock removal. Table to right of bar graph indicates subjects in good health (g); presence of health condition: a = arterial disease or peripheral vascular disease, $v=$ venous insufficiency, $h=$ high blood pressure, $d=$ diabetic, $z=$ other condition $\left(z_{1}=\right.$ heart arrhythmia, $z_{2}=$ lifetime antibiotics because of residual limb infection, $z_{3}=$ renal failure and dialysis, $z_{4}=$ congestive heart failure). $A$ "-" indicates that ambulatory strain-gauge plethysmography testing was not conducted. People who were overweight $(25<$ body mass index $\leq 30)(0)$ and obese (body mass index > 30) (O) are indicated in "O" column, and smokers (s) are indicated in "S" column. Reasons for amputation (R) include trauma (t), vascular (arterial) disease (vd), spina bifida (b), Larsson syndrome (L), and cancer (c).

but opposite in direction to those from removing the sock. We also explored how the results related to subject health.

We defined residual-limb fluid volume change as the measurement made with the bioimpedance analysis instrument used. Bioimpedance measures electrical resistance in soft tissue, and because fluid is such a dominant constituent and is of relatively low resistance, fluid volume dominates the measurement. Thus, we considered it appropriate to use the term "residual-limb fluid volume change" to describe the result from the bioimpedance measurement.

Though the exact relationship between residual-limb fluid volume change measured using bioimpedance analysis and total residual-limb volume change is not known, we can summarize reports from the literature on total limb fluid volume change. In eight transtibial amputee subjects with mature limbs, investigators reported median 
absolute value diurnal volume changes of 0.4 percent and median absolute volume changes over 5- to 25-week intervals of 1.3 percent [2] using an optical scanner. For six subjects assessed at 2-week intervals, median absolute limb-volume changes were 1.2 percent [19]. Fernie and Holliday indicated that transtibial amputee subjects had difficulty donning the socket when residual-limb volume increased by 3 to 5 percent [32]. Volume reductions during the postoperative period (12-18 mo after amputation [33]) have been recorded in numerous studies [32,34-37] and, as expected, were typically much larger than those measured in mature limbs listed previously, ranging up to 35 percent over several months. Median absolute fluid volume changes measured in the present study for sock addition or sock removal were 0.8 percent. Thus, the magnitude of fluid volume change measured with bioimpedance analysis in the present study was in the range of limb fluid volume changes reported in the literature for diurnal and shorter term time intervals measured using other measurement modalities. However, the actual quantitative correspondence between limb fluid volume and total limb volume remains to be determined.

\section{Sources of Error in Experiments}

The electrode configuration used in this study measured only from the proximal and mid-limb region of the residual limb, between the voltage-sensing electrodes (Figure 1). The distal end of the limb was not monitored. Measurement from only a portion of the residual limb is a limitation of the electrode configuration used. Thus, distal swelling, if it occurred during this study, was not included in the bioimpedance measurement. However, the proximal and central regions were considered of clinical relevance since for a properly fit prosthesis, this is where much of the mechanical support is provided by the socket for weight bearing. Oversized sockets may cause distal end bearing and thus minimal mechanical support in the proximal and central regions. It is unknown how excessive distal end bearing would affect bioimpedance results. In the present study, we made sure subjects did not distal end bear excessively, i.e., greater than approximately 10 percent of their body weight. We did not include bioimpedance data from time points that we expected were strongly affected by factors other than residual-limb fluid volume change. For example, we did not consider fluid-volume differences between sitting and standing because different tensions could have been induced in the skin between the electrodes for the two conditions. Inconsistent skin tension might have affected electrode positions and thus the bioimpedance measured. We only compared data from different time points within a session collected during a consistent posture, i.e., standing with equal weight bearing. We also calculated limb fluid volume change over the course of a 10 min interval after the prosthesis was doffed, with the subject sitting still in a chair with the limb in a dependent (downward) position.

\section{Application to Clinical Treatment}

There was a lot of variability in the bioimpedance results from different subjects. Though we added the same sock thickness on each subject, we did not see the same amount of fluid volume change or percentage fluid volume changes across the population. Thus we cannot set definitive quantitative rules of how a single sock (Soft Sock 1-ply) added to the residual limb affects a person's limb-fluid volume. We suggest two possible reasons why fluid volume change was not consistent across all subjects. First, subjects' initial socket fits were different. For some subjects, part of the added sock may have filled local vacant spaces within the socket, adding localized rather than uniform pressure to limb soft tissues, while for other subjects, sock addition may have caused a moderate or large pressure increase over the entire residuum. Second, subjects had different limb soft tissue mechanical consistencies. Thus, even though sock thickness was the same, the stress induced on the soft tissues and the fluid volume changes induced were not the same for all subjects.

Fluid-volume change for sock addition was not equal to fluid-volume change for sock removal. This result suggests that care must be taken during fitting sessions. Adding and removing socks on a patient during a fitting session might change the patient's limb fluid volume. In other words, changes in limb fluid volume might be accentuated by the history of sock change. The practitioner needs to be mindful of these changes during fitting. Adding a very thick sock is discouraged, particularly for patients with compromised arterial drive, with diabetes, or who are obese or smoke, because this sock may cause much limb fluid volume reduction that will not be easily recovered.

Differences in percentage fluid volume change from sock addition and sock removal seem to be linked with the subject's vascular condition. Subjects with arterial vascular complications tended to lose fluid volume upon sock addition and recover minimally or tended to continue to reduce in fluid volume when the sock was removed (group 1, group 2 with high AR ratios; Figure 4). These subjects 
likely had reduced transport from the arterial vasculature into the interstitial space. The net effect was a loss in limbfluid volume over the course of the test session.

Because ASGP measurements were made on only 19 of the 28 participants, we cannot determine if venous insufficiency dominated one group. Further, venous insufficiency, particularly if it results from local trauma, can be present in one limb but not the other; thus, ASGP results from the contralateral limb do not necessarily reflect the residual limb. The result that some subjects in group 2 with low AR ratios had venous insufficiency but not arterial disease is consistent with the interpretation that subjects who had poor venous flow experienced low residual-limb fluid volume loss upon sock addition. However, further research is needed to verify this interpretation and to determine if the presence of venous disease on limb fluid volume change offsets the effects of arterial disease.

Unexpectedly, the group 4 subject's fluid volume enlarged upon sock addition and reduced upon sock removal. This subject may have been at high risk of venous occlusion, as supported by ASGP results indicating venous insufficiency. The added sock may have increased interface pressures, particularly over the posterior vasculature, and as a result occluded venous return but not arterial inflow. The net effect may have been to increase limb fluid volume when a sock was added and reduce it when the sock was removed. While this phenomenon might have contributed to the observed result, another possible contribution was determined subsequent to the study. We determined that this subject had metal orthopedic hardware within his limb. The hardware may have altered electrical current distribution through the soft tissues and thus affected the bioimpedance data we recorded, confounding interpretation.

Smoking and obesity are considered to have a detrimental effect on the vasculature, causing a loss of vessel compliance [38-39]. A loss of vascular compliance would be expected to reduce arterial drive, resulting in a reduction in arterial to interstitial fluid transport. This phenomenon might explain why smokers and subjects with obesity, in general, underwent a reduction in limb fluid volume over the course of the test session (i.e., group 1 subjects or group 2 subjects with high AR ratios; Figure 4). Similarly, presence of diabetes is expected to affect the vasculature and reduce arterial drive. Loss of pressure-induced vasodilation has been demonstrated in people with diabetes [4042]. This phenomenon might explain why subjects with diabetes, in general, also underwent a reduction in limb fluid volume over the test session (Figure 4). A next step is to quantify severity of diabetes, for example by using glycosylated hemoglobin testing (HbA1c), and investigate a correlation with residual-limb fluid-volume change.

High blood pressure, in general, would be expected to increase arterial-to-interstitial fluid transport and thus induce edema over the session, but presence of high blood pressure in our subjects did not appear to be related to bioimpedance results. Because of the many different physiological mechanisms and factors contributing to elevated blood pressure, the manifestation of peripheral edema is not universal in people diagnosed with hypertension. Additionally, many subjects with diagnosis of hypertension are treated with medications, which might have mitigated the edema-prone effects of high blood pressure. Further, high blood pressure typically presents with other health problems, and these other problems might have complicated impact on limb fluid volume change.

Presence of a heart condition and dialysis treatment would be expected to affect limb fluid transport as well. There were too few subjects with these conditions in the present study to draw meaningful conclusions about their impact on limb fluid volume control after sock addition or removal. A separate and larger study targeting subject populations with these conditions is needed.

Because of the lack of a one-to-one correlation between vascular status and bioimpedance results, other factors besides vascular status likely affected limb fluid volume control. The results from the present study indicate that the subject's vascular condition is a relevant factor, but more research is needed to identify and understand how other factors affect limb fluid volume control. Socket fit might affect the results because it affects local pressure and shear stress changes induced from sock addition. The relationships between socket design, interface stress distribution, and limb fluid volume change are currently unknown. In addition, it would be helpful to identify the level of importance of each factor. Such an effort would help practitioners and patients better manage limb fluid volume and its effect on socket fit.

Interestingly, three of the five subjects in the present study who demonstrated limb fluid volume increases over the session were deemed in good health as per the vascular tests and other information used here (Figure 4). Nonamputated limbs of healthy people also demonstrated this result [43-45], as did the contralateral limb of a person with unilateral amputation [46]. On normal limbs, fluid volume increases over time are considered a result 
of gravity pulling fluid distally toward the feet during standing and walking. At night, while supine, limb fluid volume reduces and returns to normal. Because of the continual stress applied by the prosthetic socket on the residual limb during standing and walking and thus the increase in interstitial fluid pressure, people with limb amputation would be expected to undergo limb fluid volume decreases over time, not increases. Why then did our three healthy subjects experience limb fluid volume increases? It may be that the effects of gravity toward increasing limb fluid volume outweighed the influence of socket interface pressures to decrease it. Two of the three healthy subjects and four of the five subjects in group 3 were overweight. People who are overweight tend to build up fluid in their lower limbs. Increased fat may increase pressure in the legs and contribute to edema. It is also possible that the subjects' physiological control systems adapted since amputation so as to induce limb fluid volume increases during activity, consistent with normal nonamputated limbs. Neural and endocrine factors also may have played a role. Whatever the source, the result that limb fluid volume increases over time overshadowed the influence of sock addition and removal for some of the healthy subjects is surprising and worthy of further investigation.

The subject who underwent a different protocol demonstrated directions of fluid volume change and AR ratio results consistent with group 2 subjects. However, her absolute volume changes were much larger (3.4\% loss upon sock addition; $2.1 \%$ gain upon sock removal) than the other subjects. We suspect this difference reflected her adding/removing a thicker sock (3-ply vs 1-ply). Dependence of limb volume change on thickness of the added sock should be investigated.

\section{Application to Volume Control Devices}

The results from this investigation point to caution in implementing automatic volume control systems. If material is to be added into the socket to accommodate limb volume loss, for example fluid-filled or air-filled inserts, then care must be taken to ensure that the amount of fluid or air added does not further reduce limb volume. Some subjects might not recover fluid that is displaced out of the residual limb. Products that adjust their size over time according to the pressure they put on the limb, e.g., the Active Volume Control System (Simbex; Lebanon, New Hampshire), may help allow recovery of fluid into the residual limb. However, this interpretation is conjecture and needs research investigation. It would also be worthwhile to investigate the long-term effects of these technologies on residual-limb fluid volume and compare with results using traditional accommodation strategies (e.g., sock changes).

Subjects who showed a decrease in limb fluid volume without recovery when the sock was removed (group 1 subjects, group 2 subjects with high AR ratios) may be good candidates for elevated vacuum. Elevated vacuum applies negative pressure to the socket, unlike the positive pressure applied here by the added socks. Elevated vacuum might elevate limb fluid volume during walking [47-52]. If timed such that greater vacuum was applied during activity, elevated vacuum may help stabilize limb fluid volume over time. However, elevated vacuum sockets are typically smaller than pin suspension sockets, which may counter this beneficial effect. It would be interesting to investigate the long-term effects of elevated vacuum and other compensation strategies on residual-limb fluid volume measured by bioimpedance.

\section{Bioimpedance Analysis as Diagnostic Tool}

The bioimpedance analysis instrumentation implemented in this research potentially could be extended into a diagnostic tool to help a practitioner determine if an accommodation strategy or volume control algorithm is needed and prescribe it on a patient-by-patient basis. Who needs elevated vacuum and who does not? What volume control protocol should be used and why? We would suspect that group 1 and group 2 subjects with high AR ratios are good candidates for elevated vacuum and would do better with than without it. This interpretation is still conjecture at this point and will need to be tested in a controlled clinical study with a large sample before it is applied to clinical practice.

Subjects who showed an increase in limb fluid volume over time that outweighed the influence of sock addition and removal might be prone to edema and thus probably would not be good candidates for elevated vacuum. We suspect that elevated vacuum would worsen their diurnal limb fluid volume increase, particularly for patients with slow overall fluid transport in their residual limb. This interpretation is conjecture as well and needs careful clinical testing.

Over time, subjects in group 2 with arterial disease would be expected to increase their AR ratios and then eventually move into group 1 (negative AR ratio). Alternatively, if they developed venous disease, they would be 
expected to decrease their AR ratios and move toward group 3. At this point, we have not collected data over long enough time intervals to be able to determine if these trends occur.

\section{Future Research}

In addition to the investigations we have suggested, future research needs to be directed toward longer-term monitoring. What are residual-limb fluid volume changes over the course of a day, and does sock addition cause a long-lasting alteration in limb fluid volume? How do results relate to subject comfort and satisfaction with the prosthetic prescription? How does activity influence the results? What is the influence of patient lifestyle changes, treatments, medications, diet, or other variables on limb fluid balance? If these questions are answered and if a relationship between long-term and short-term fluid volume changes can be established, then limb fluid volume change technology could be used as a tool to help a practitioner characterize a patient's physiological capability to control limb fluid volume, maintain health, and facilitate prosthetic prescription.

\section{CONCLUSIONS}

Residual-limb fluid volume losses induced by adding a 1-ply polyester sock were not equal to gains induced by removing the 1-ply sock. Practitioners should be mindful of this result during fitting. Recent history of sock use may affect the current fit of the prosthesis. Participants who had arterial disease, were obese, or were smokers tended to experience fluid volume losses for both sock addition and sock removal, or they experienced relatively high fluid volume losses for sock addition and relatively low fluid volume gains for sock removal. Several participants in good health experienced limb fluid volume gains over the course of the session. Interestingly, nondisabled people experience fluid volume gains in their lower limbs over the day. Bioimpedance analysis could potentially be extended into a diagnostic tool to help practitioners evaluate a patient's limb fluid volume control and select an accommodation strategy.

\section{ACKNOWLEDGMENTS}

Author Contributions:

Study concept and design: J. E. Sanders.
Acquisition of data: D. S. Harrison, K. J. Allyn, T. R. Myers. Analysis and interpretation of data: J. E. Sanders, K. J. Allyn, T. R. Myers, E. C. Tsai.

Drafting of manuscript: J. E. Sanders.

Critical revision of manuscript for important intellectual content: K. J. Allyn, M. A. Ciol, E. C. Tsai.

Statistical analysis: M. A. Ciol.

Administrative, technical, or material support: D. S. Harrison, K. J. Allyn.

Financial Disclosures: The authors have declared that no competing interests exist.

Funding/Support: The material was based on work supported by the Orthotic and Prosthetic Education and Research Foundation within the American Academy of Orthotists and Prosthetists and the National Institutes of Health (grant R01HD60585).

Additional Contributions: Timothy R. Myers is now with Newberg Roadhouse LLC.

Institutional Review: Human subjects approval from a University of Washington Internal Review Board was received and informed consent was obtained before any study procedures were initiated.

Participant Follow-Up: The authors plan to inform participants of the publication of this study if they requested to be informed.

\section{REFERENCES}

1. Lilja M, Johansson S, Oberg T. Relaxed versus activated stump muscles during casting for trans-tibial prostheses. Prosthet Orthot Int. 1999;23(1):13-20. PMID:10355639

2. Sanders JE, Zachariah SG, Jacobsen AK, Fergason JR. Changes in interface pressures and shear stresses over time on trans-tibial amputee subjects ambulating with prosthetic limbs: Comparison of diurnal and six-month differences. J Biomech. 2005;38(8):1566-73. PMID:15958212 http://dx.doi.org/10.1016/j.jbiomech.2004.08.008

3. Goldstein B, Sanders J. Skin response to repetitive mechanical stress: A new experimental model in pig. Arch Phys Med Rehabil. 1998;79(3):265-72. PMID:9523777 http://dx.doi.org/10.1016/S0003-9993(98)90005-3

4. Van Loan MD, Withers P, Matthie J, Mayclin PL. Use of bioimpedance spectroscopy to determine extracellular fluid, intracellular fluid, total body water, and fat-free mass. Basic Life Sci. 1993;60:67-70. PMID:8110166

5. Organ LW, Bradham GB, Gore DT, Lozier SL. Segmental bioelectrical impedance analysis: Theory and application of a new technique. J Appl Physiol. 1994;77(1):98-112. PMID:7961281

6. Fuller NJ, Hardingham CR, Graves M, Screaton N, Dixon AK, Ward LC, Elia M. Predicting composition of leg sections with anthropometry and bioelectrical impedance analysis, using magnetic resonance imaging as reference. Clin Sci (Lond). 1999;96(6):647-57. PMID:10334971 http://dx.doi.org/10.1042/CS19980376 
7. Salinari S, Bertuzzi A, Mingrone G, Capristo E, Scarfone A, Greco AV, Heymsfield SB. Bioimpedance analysis: A useful technique for assessing appendicular lean soft tissue mass and distribution. J Appl Physiol. 2003;94(4):1552-56. PMID:12626475

8. Dittmar M. Reliability and variability of bioimpedance measures in normal adults: Effects of age, gender, and body mass. Am J Phys Anthropol. 2003;122(4):361-70. PMID:14614757 http://dx.doi.org/10.1002/ajpa.10301

9. Andreoli A, Melchiorri G, De Lorenzo A, Caruso I, Sinibaldi Salimei P, Guerrisi M. Bioelectrical impedance measures in different position and vs dual-energy X-ray absorptiometry (DXA). J Sports Med Phys Fitness. 2002;42(2):186-89. PMID:12032414

10. De Vries PM, Meijer JH, Vlaanderen K, Visser V, Oe PL, Donker AJ, Schneider H. Measurement of transcellular fluid shift during haemodialysis. Part 2. In vitro and clinical evaluation. Med Biol Eng Comput. 1989;27(2):152-58. PMID:2601434

11. Zhu F, Schneditz D, Wang E, Martin K, Morris AT, Levin NW. Validation of changes in extracellular volume measured during hemodialysis using a segmental bioimpedance technique. ASAIO J. 1998;44(5):M541-45. PMID:9804490 http://dx.doi.org/10.1097/00002480-199809000-00045

12. Shulman T, Heidenheim AP, Kianfar C, Shulman SM, Lindsay RM. Preserving central blood volume: Changes in body fluid compartments during hemodialysis. ASAIO J. 2001;47(6):615-18. PMID:11730198 http://dx.doi.org/10.1097/00002480-200111000-00009

13. Donadio C, Consani C, Ardini M, Bernabini G, Caprio F, Grassi G, Lucchesi A, Nerucci B. Estimate of body water compartments and of body composition in maintenance hemodialysis patients: Comparison of single and multifrequency bioimpedance analysis. J Ren Nutr. 2005;15(3): 332-44. PMID:16007563 http://dx.doi.org/10.1016/j.jrn.2005.04.001

14. Fuller NJ, Hardingham CR, Graves M, Screaton N, Dixon AK, Ward LC, Elia M. Predicting composition of leg sections with anthropometry and bioelectrical impedance analysis, using magnetic resonance imaging as reference. Clin Sci (Lond). 1999;96(6):647-57. PMID:10334971 http://dx.doi.org/10.1042/CS19980376

15. Segal KR, Burastero S, Chun A, Coronel P, Pierson RN Jr, Wang J. Estimation of extracellular and total body water by multiple-frequency bioelectrical-impedance measurement. Am J Clin Nutr. 1991;54(1):26-29. PMID:2058583

16. Wotton MJ, Thomas BJ, Cornish BH, Ward LC. Comparison of whole body and segmental bioimpedance methodologies for estimating total body water. Ann N Y Acad Sci. 2000;904:181-86. PMID:10865733 http://dx.doi.org/10.1111/j.1749-6632.2000.tb06444.x
17. Armstrong LE, Kenefick RW, Castellani JW, Riebe D, Kavouras SA, Kuznicki JT, Maresh CM. Bioimpedance spectroscopy technique: Intra-, extracellular, and total body water. Med Sci Sports Exerc. 1997;29(12):1657-63. PMID:9432101 http://dx.doi.org/10.1097/00005768-199712000-00017

18. Miyatani M, Kanehisa H, Masuo Y, Ito M, Fukunaga T. Validity of estimating limb muscle volume by bioelectrical impedance. J Appl Physiol. 2001;91(1):386-94. PMID:11408456

19. Zachariah SG, Saxena R, Fergason JR, Sanders JE. Shape and volume change in the transtibial residuum over the short term: Preliminary investigation of six subjects. J Rehabil Res Dev. 2004;41(5):683-94. PMID:15558398 http://dx.doi.org/10.1682/JRRD.2003.10.0153

20. Keys A, Fidanza F, Karvonen MJ, Kimura N, Taylor HL. Indices of relative weight and obesity. J Chronic Dis. 1972; 25(6):329-43. PMID:4650929 http://dx.doi.org/10.1016/0021-9681(72)90027-6

21. Mozumdar A, Roy SK. Method for estimating body weight in persons with lower-limb amputation and its implication for their nutritional assessment. Am J Clin Nutr. 2004; 80(4):868-75. PMID:15447892

22. Grimnes S, Martinsen OG. Bioimpedance and bioelectricity basics. 2nd ed. Amsterdam (the Netherlands): Elsevier Ltd; 2008.

23. De Lorenzo A, Andreoli A, Matthie J, Withers P. Predicting body cell mass with bioimpedance by using theoretical methods: A technological review. J Appl Physiol. 1997; 82(5):1542-58. PMID:9134904

24. Fenech M, Jaffrin MY. Extracellular and intracellular volume variations during postural change measured by segmental and wrist-ankle bioimpedance spectroscopy. IEEE Trans Biomed Eng. 2004;51(1):166-75. PMID:14723506 http://dx.doi.org/10.1109/TBME.2003.820338

25. Clement D, editor. Inter-society consensus for the management of PAD. Diagnosis and evaluation of peripheral artery disease. Discovery London and TASC II; 2008. Available from:

http://www.tasc-2-pad.org/upload/SSRubriqueProduit/ Fichier2/963.pdf.

26. Nicolaides AN; Cardiovascular Disease Educational and Research Trust; European Society of Vascular Surgery; The International Angiology Scientific Activity Congress Organization; International Union of Angiology; Union Internationale de Phlebologie at the Abbaye des Vaux de Cernay. Investigation of chronic venous insufficiency: A consensus statement (France, March 5-9, 1997). Circulation. 2000; 102(20):E126-63. PMID:11076834

27. Adragao T, Pires A, Branco P, Castro R, Oliveira A, Nogueira C, Bordalo J, Curto JD, Prata MM. Ankle-brachial index, vascular calcifications and mortality in dialysis 
patients. Nephrol Dial Transplant. 2012;27(1):318-25. PMID:21551082

http://dx.doi.org/10.1093/ndt/gfr233

28. Potier L, Abi Khalil C, Mohammedi K, Roussel R. Use and utility of ankle brachial index in patients with diabetes. Eur J Vasc Endovasc Surg. 2011;41(1):110-16.PMID:21095144 http://dx.doi.org/10.1016/j.ejvs.2010.09.020

29. Struckmann JR. Assessment of the venous muscle pump function by ambulatory strain gauge plethysmography. Methodological and clinical aspects. Dan Med Bull. 1993; 40(4):460-77. PMID:8222765

30. Medicare region C durable medical equipment prosthetics orthotic supplier (DMEPOS) manual. Columbia (SC): Palmetto GBA; 2005. p. 53.5-6.

31. Scott TE, LaMorte WW, Gorin DR, Menzoian JO. Risk factors for chronic venous insufficiency: A dual case-control study. J Vasc Surg. 1995;22(5):622-28. PMID:7494366 http://dx.doi.org/10.1016/S0741-5214(95)70050-1

32. Fernie GR, Holliday PJ. Volume fluctuations in the residual limbs of lower limb amputees. Arch Phys Med Rehabil. 1982; 63(4):162-65. PMID:7082139

33. American Academy of Orthotists and Prosthetists. Postoperative management of the lower extremity amputee: Official findings of the Consensus Conference. J Prosthet Orthot. 2004;16(3S):2-14.

34. Wilson AB, Jr, Schuch CM, Nitschke RO. A variable volume socket for below-knee prostheses. Clin Prosthet Orthot. 1987;11(1):11-19.

35. Lilja M, Öberg T. Proper time for definitive transtibial prosthetic fitting. J Prosthet Orthot. 1997;9(2):90-95. http://dx.doi.org/10.1097/00008526-199700920-00009.

36. Liedberg E, Hommerberg H, Persson BM. Tolerance of early walking with total contact among below-knee amputees-A randomized test. Prosthet Orthot Int. 1983;7(2):91-95. PMID:6622240

37. Golbranson FL, Wirta RW, Kuncir EJ, Lieber RL, Oishi C. Volume changes occurring in postoperative below-knee residual limbs. J Rehabil Res Dev. 1988;25(2):11-18. PMID:3361456

38. Kool MJ, Hoeks AP, Struijker Boudier HA, Reneman RS, Van Bortel LM. Short- and long-term effects of smoking on arterial wall properties in habitual smokers. J Am Coll Cardiol. 1993;22(7):1881-86.PMID:8245343

http://dx.doi.org/10.1016/0735-1097(93)90773-T

39. Toto-Moukouo JJ, Achimastos A, Asmar RG, Hugues CJ, Safar ME. Pulse wave velocity in patients with obesity and hypertension. Am Heart J. 1986;112(1):136-40.

PMID:3728268

http://dx.doi.org/10.1016/0002-8703(86)90691-5

40. Koïtka A, Abraham P, Bouhanick B, Sigaudo-Roussel D, Demiot C, Saumet JL. Impaired pressure-induced vasodilation at the foot in young adults with type 1 diabetes. Diabe- tes. 2004;53(3):721-25. PMID:14988257

http://dx.doi.org/10.2337/diabetes.53.3.721

41. McLellan K, Petrofsky JS, Zimmerman G, Lohman E, Prowse M, Schwab E, Lee S. The influence of environmental temperature on the response of the skin to local pressure: The impact of aging and diabetes. Diabetes Technol Ther. 2009;11(12):791-98. PMID:20001680

http://dx.doi.org/10.1089/dia.2009.0097

42. Fromy B, Sigaudo-Roussel D, Gaubert-Dahan ML, Rousseau P, Abraham P, Benzoni D, Berrut G, Saumet JL. Aging-associated sensory neuropathy alters pressureinduced vasodilation in humans. J Invest Dermatol. 2010; 130(3):849-55. PMID:19727122 http://dx.doi.org/10.1038/jid.2009.279

43. Ludbrook J, Loughlin J. Regulation of volume in postarteriolar vessels of the lower limb. Am Heart J. 1964;67:493-507. PMID:14138811 http://dx.doi.org/10.1016/0002-8703(64)90096-1

44. Stick C, Stöfen P, Witzleb E. On physiological edema in man's lower extremity. Eur J Appl Physiol Occup Physiol. 1985;54(4):442-49. PMID:4065133 http://dx.doi.org/10.1007/BF02337192

45. Stick C, Jaeger H, Witzleb E. Measurements of volume changes and venous pressure in the human lower leg during walking and running. J Appl Physiol. 1992;72(6):2063-68. PMID:1629057

46. Sanders JE, Harrison DS, Allyn KJ, Myers TR. Clinical utility of in-socket residual limb volume change measurement: Case study results. Prosthet Orthot Int. 2009;33(4): 378-90. PMID:19961297 http://dx.doi.org/10.3109/03093640903214067

47. Caspers CA. Hypobarically-controlled artificial limb for amputees. United States patent US 5,549,709. 1996 Aug 27.

48. Street GM. Vacuum suspension and its effect on the limb. Orthop Tech. 2007;4:1-4.

49. Board WJ, Street GM, Caspers C. A comparison of transtibial amputee suction and vacuum socket conditions. Prosthet Orthot Int. 2001;25(3):202-9.PMID:11860094 http://dx.doi.org/10.1080/03093640108726603

50. Goswami J, Lynn R, Street G, Harlander M. Walking in a vacuum-assisted socket shifts the stump fluid balance. Prosthet Orthot Int. 2003;27(2):107-13. PMID:14571940 http://dx.doi.org/10.1080/03093640308726666

51. Gerschutz MJ, Denune JA, Colvin JM, Schober G. Elevated vacuum suspension influence on lower limb amputee's residual limb volume at different vacuum pressure settings. J Prosthet Orthot. 2010;22(4):252-56. http://dx.doi.org/10.1097/JPO.0b013e3181f903df

52. Sanders JE, Harrison DS, Myers TR, Allyn KJ. Effects of elevated vacuum on in-socket residual limb fluid volume: Case study results using bioimpedance analysis. J Rehabil 
JRRD, Volume 49, Number 2, 2012

Res Dev. 2011;48(10):1231-48. [PMID: 22234667]

http://dx.doi.org/10.1682/JRRD.2010.11.0219

Submitted for publication February 18, 2011. Accepted in revised form August 11, 2011.

This article and any supplementary material should be cited as follows:

Sanders JE, Harrison DS, Allyn KJ, Myers TR, Ciol MA, Tsai EC. How do sock ply changes affect residual-limb fluid volume in people with transtibial amputation? J Rehabil Res Dev. 2012;49(2):241-56. http://dx.doi.org/10.1682/JRRD.2011.02.0022

ResearcherID: Joan E. Sanders, PhD: E-8204-2011

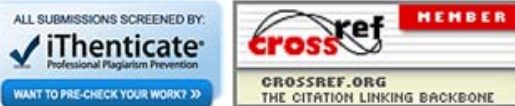

